\title{
Trade-off between Energy Consumption and Transmission Rate in Mobile Ad-Hoc Network
}

\author{
Ashraf Al Sharah ${ }^{1}$, Mohammad Alhaj ${ }^{2}$ \\ Computer Engineering Department \\ Al-Ahliyya Amman University, Amman, Jordan
}

\author{
Firas Al Naimat ${ }^{3}$ \\ Medical Engineering Department \\ Al-Ahliyya Amman University, Amman, Jordan
}

\begin{abstract}
Mobile Ad-Hoc Networks are decentralized systems of mobile nodes where each node is responsible for computing and retaining the routing and topology details. The autonomous nature of the nodes stresses more on the way of handling power consumptions. This raises a concern about how to improve the power efficiency that leads to a better battery life of the mobile nodes. It is important to balance between power and transmission rates to improve the network lifetime and reduce the sudden failure of the nodes. In this paper, a new transmission power control-based scheme is proposed that allows the mobile nodes to achieve a tradeoff between balancing transmission rate and power consumption. Through defining and updating two tables for every node that contain the average transmission rate for the neighboring nodes and number of times the neighboring node is used for data transmission. We validate our proposed scheme using several test cases.
\end{abstract}

Keywords-Coalition; MANETs; power-aware routing; power consumption; transmission power control-based; transmission rate

\section{INTRODUCTION}

Mobile Ad-Hoc Networks (MANETs) are wireless networks where a group of mobile nodes operate independently in order to configure and hold their ad-hoc network routing and topology information. MANETs do not rely on present infrastructure, but instead each mobile node broadcast messages to the neighboring nodes. They are popular in different military and domestic applications such as ship-to-ship, law enforcement communications and ecological monitoring [1].

MANET provides a decentralized control with uncertain connections among nodes and a rapid mobility of hosts. The mobile nodes act as routers, monitors and decision makers. It has a limited availability of resources where the processing power, memory capacity, battery performance, and bandwidth relies on the mobile nodes of the network. MANET provides vulnerable and insecure operating environment; and it suffers from low transmission rate, data overhead according to not choosing the optimal bandwidth and power consumption. Since its topology varies dynamically and frequently, mobile nodes may appear and disappear abruptly.

Cooperation between mobile nodes in MANETs is important to assure the best performance and utilization. For that purpose, game theory is used to solve interactions between nodes in the network. Game theory is the best practice to study situations of mutual interest among mobile nodes, solve conflict between them and find the suitable actions for an individual node [2]. There are two kinds of game theory: a) Non-cooperative game where the strategic choices of each node are chosen based his own interest without any commitment with the others; and b) Cooperative game where nodes have mutual interest with each other and can make binding commitments. All nodes in this paper are cooperative, have mutual interests and exchange transmission details between the neighboring nodes.

The efficient utilization of power is a major concern in MANETs where nodes in the network are constrained by power consumption and computational resource. The battery life time determines the mobility time of a node in a network. With the increasing of mobility time, the power consumption increases and the remaining node battery life decreases. The decision of route selection decision is becoming more challenging than just selecting the route with the lowest power consumption [3][4]. Using the power-aware routing to examine the power consumption of the nodes when making routing decisions improves the performance of routing in MANETs [5].

Transmission rate is another concern in optimizing the performance of routing in MANETs. The transmission rate in MANETs is normally unstable and keeps changing during the time. In order to assure the stability of transmission rates, it is important to select the right rate between the neighboring nodes for data exchanging and avoid data overhead or data loss in the transmission channel. A previous research in [6] proposes a mechanism called Hand-shake Rate Adaptation (HRA) that avoids wrong selections of transmission rate in MANET by specifying the average transmission rate between its mobile nodes. Mobile nodes can select the appropriate transmission rates to ensure that bandwidth requirements are met. The mechanism adopts a decentralized approach where nodes are involved in calculating transmission rate for the neighbors, therefor by knowing the neighbors' transmission rates, individual node is able to select the proper rates. Mobile nodes in MANETs also have different transmission power based on the amount of transmitted data. Choosing a wrong transmission rate increases the power consumption and affects the efficiency of its power utilization [7][8][9][10].

A literature survey in [11] summarizes 51 reviewed papers about power-efficient routing schemes in MANETs based on topology information and protocol operations. The survey categorizes the routing schemes into six approaches: 
1) Link state-based where each node finds the shortest path through exchanging the routing table information with the neighbors.

2) Source initiated-based where the sending node perform the discovery procedure when it is necessary to find the path to the destination node.

3) Transition power control-based which focuses on adjusting the transmission power between the source node and the destination node.

4) Load balancing-based which focuses on balancing energy usage among all nodes by choosing a routing path that includes previously unused mobile nodes instead of choosing the shortest path.

5) Location-based which selects the routing path between the source and destination nodes using the geographic position techniques such as global positioning system GPS or location aided routing (LAR) [12].

6) Multicast-based which broadcasts the data from one source to all destinations within a multicast group.

In this paper, we propose a scheme based on transmission power control-based approach to provide a balance between the transmission rates and power consumption of mobile nodes. The scheme uses a cascaded single-hop to discover the utilization and the average transmission rate of the neighboring node. Each node maintains two tables: one contains number of times that a node utilizes the neighbor nodes in data transmission, and another table contains the assigned average transmission rate between each node. Based on the two tables, nodes can select the suitable neighbor node in transmitting data. The scheme increases the life time of the network and reduces the rate of failing nodes.

The paper is organized as follows, Section II presents the background and related work; Section III presents the proposed model; Section IV presents the proposed algorithm; Section V presents the simulation and results; Section VI provides conclusion and future work.

\section{BACKGROUND AND RELATED WORK}

MANET is a decentralized type of networks where each node contributes in forwarding data to the neighbor nodes [13]. MANET does not rely on routers, access points or other communication infrastructure; instead data is transmitted through the neighboring nodes based on defined routing algorithm. Several applications are available for simulating the behavior of MANET. A common one is called Network Simulator NS-2 [14]. NS-2 is an open source simulation tool which provides a discrete event simulating for TCP, routing, and multicast protocols over wired and wireless networks.

The transmission power control-based approach is introduced in a survey [11] with eight reviewed papers that are similar to our proposed scheme. Chang and Tassiulas in [15] propose a flow augmentation routing (FAR) protocol to balance the traffic between the nodes by selecting the routes and the corresponding power until the batteries of the nodes drain-out is maximized. This allows defining the optimal levels of transmission power and the optimal route. $\mathrm{Li}$ et al. in [16] propose an online max-min power-aware routing protocol (OMM) that uses Dijkstra's algorithm to find the optimal path for a given source-destination pair. An empirical competitive ratio is developed to optimize the lifetime of MANET through minimizing the consumed power and maximizing the minimal residual energy of the node. Doshi and Brown in [17] introduce a minimum energy routing (MER) scheme to select a path that minimizes the energy required for routing a packet from the source to the destination. The path is selected by finding the link cost in terms of the packet transmission energy, the energy required for route discovery and the energy consumed to maintain routes. Avudainayagam et al. in [18] propose a device- and energy-aware routing (DEAR) protocol for heterogeneous wireless ad hoc network with two types of nodes: externallypowered nodes and battery-powered nodes. Based on the energy and the device awareness of the routing protocol, the externally-powered nodes have higher priorities of the transmission and perform more routing functions. Rishiwal et al. in [19] propose a power-aware routing (PAR) protocol to improve the network lifetime through selecting a less congested and more stable routes for data transmission. The selection is based on three metrics: the overall energy of a path, the status of battery lifetime and the type of data to transfer. Yanez-Marquez et al. in [20] introduce a minimum spanning tree (MST) to select the optimal routing by calculating the minimum spanning tree using a low complexity algorithm based on the binary decision diagrams (BDDs). Lalitha and Rajesh in [21] propose an hoc Ondemand Distance Vector Range Routing (AODV_RR) that improves the overall energy consumption of MANETs by reducing the communication overhead. Finally, Katiravan et al. in [22] propose an Energy Efficient Link Aware Routing with Power Control (ELRPP) to select the optimal routing using three metrics: residual energy, signal to noise ratio (SNR) and link quality. A comparison between the proposed scheme and the previous routing schemes within the same category is presented in Section V.

There are also recent researches which have been devoted great efforts on power in mobile ad-hoc networks. Kannan and Rajaram in [23] design and develop strategy of QoS aware power efficient multicast routing protocol (QoS-PEMRP) suitable for mobile nodes. The development is based on QoS metrics such as average group delivery ratio, average power consumption and average delay. Papageorgiou et al. [24] propose an energy-efficient multicasting algorithm that select the optimal energy-efficient set of nodes for multicasting. Three parameters are considered: the node residual energies, the transmission powers, and the set of covered nodes. Khan et al. in [25] focuses in reducing the coast of coding mechanism by reducing the size of data used for permutation. The idea of the mechanism is that the source permutes global encoding vectors only and not considering the whole message symbols to achieve better energy consumption. Rahman in [26] proposes an algorithm that selects the route over earlier most forward within radius (MFR) method based on weighted combination of metrics of distance, velocity and battery power. The proposed algorithm improves the load balancing and increases the network performance. 
Ghode and Bhoyar in [27] introduce energy constraints in Zone Routing Protocol (ZRP) in order to make the protocol works efficiently in MANET and improves network lifetime. Hassan and Muniyandi in [28] propose a QoS-routing algorithm that supports the QoS parameters of energy and delay using Cellular Automata (CA) with Genetic Algorithm+African Buffalo Optimization (GAABO) techniques. The research aims to improve the network lifetime as well as the end-to-end delay. Lu and Zhu in [29] propose a new protocol called EDCMRA. EDCMRA is a multicast routing that maintains the energy efficiency and delay of data transmission. It uses the possible multicast trees those traced in route request process as initial input chromosomes, and uses the common subtree of the any of given two multicast tress as crossover point.

Kamboj and Sharma in [30] introduce modern solutions and models that enables multicasting. The models aim to improve the network life time. While Zhao et al. in [31] present a measure called Energy Efficiency Metric (EEM), that is the result of aggregate hop-count values and relative levels of increment of lifetime. Papanna et al. in [32] addresses the consumption of energy and maximizing the route lifetime in MANETs using the Energy Efficient and Lifetime Aware Multicast (EELAM) modeling topology and compare the results the other bench marking models like EACNS, EDCMRA. Sarkohaki et al. in [33] utilizes the artificial immune system (AIS) to improve the efficiency of the Optimized link state routing protocol (OLSR). OLSR is a mobile routing protocol that uses Dijkstra's algorithm to define the shortest route between source and destination. Ray and Turuk in [34] propose an energy conservation technique to reduce the transmission power of a node in MANETs. The technique is called Location Based Topology Control with Sleep Scheduling that allows a node to go to sleep state based on the traffic condition. Alani et al. in [35] propose a new method to improve the energy efficiency and routing performance through adapting lion optimization algorithm after specifying all possible paths in MANET. Bhatia et al. [36] propose an algorithm that is an extension of Dynamic Source Routing (DSR) routing protocol. The algorithm is used to optimize the routing bandwidth and improve the energy consumption.

In summary, it is obvious that the works above have addressed features similar to our work. The major advantage of the proposed scheme is that it achieves a tradeoff between balancing transmission rate and power consumption. The scheme algorithm is simple and developed based on cascaded single-hop discovery. The scheme algorithm selects the routing path by creating routing tables for each neighboring node. The table contains the average transmission rate for the neighboring nodes and number of times the neighboring node is used for data transmission. The proposed approach affects the overall network lifetime by increasing the battery power of the nodes and decreasing the power consumption in the network.

\section{PROPOSED NETWORK MODEL}

MANETs suffer from different challenges that affect their operations. One of these challenges is battery life time and restriction of battery power. Operations of mobile nodes in MANETs depend on the power consumption. When mobile nodes run out of battery, their ability to operate in the network traffic are affected which then affects the overall network lifetime. The lifetime of MANETs can be improved by decreasing the power consumption in the network.

The proposed model of the network is considered as a coalition repeated game, where network nodes cooperate with each other's during the repeated network life cycle. For each node in the network, a scheme of two routing tables is created. The first routing table defines the number of times the node used its neighbor for data transmission within period of time. It provides an approximate of power consumed in each node by finding how many times it's been used for data transmission. The second routing table defines the transmission rates between the node and its neighbor. The purpose of the scheme is to balance between the battery power consumption of the nodes and the transmission rates. The tables are shared with the neighboring node to guarantee fairness between nodes and control node usage in the network.

The proposed model uses cascaded single-hop routing to define the connection path between the source and destination nodes. In a cascaded single-hop routing, a sending node selects an optimal next neighboring node in a sequence of single hops. Using the routing tables at each single-hop, the sending node forwards the data to the next node based on the neighbor minimum number of time the node is used for transmission and the average rate.

Fig. 1 describes a sample of the network model of our scheme with five nodes. The scheme can be applied to any number of nodes. Each node has its own scheme where $r_{i j}$ is the average transmission rate between two neighbor nodes $i$ and $j ; t_{i j}$ is the number of times that node $i$ used node $j$ for data sending. Based on the proposed scheme, a node selects the neighbor for data transmission as follows:

average $\left(r_{i j}\right) \times$ number of times $\left(t_{i j}\right)=\min \left(r_{i j} \times t_{i j}\right)(1)$

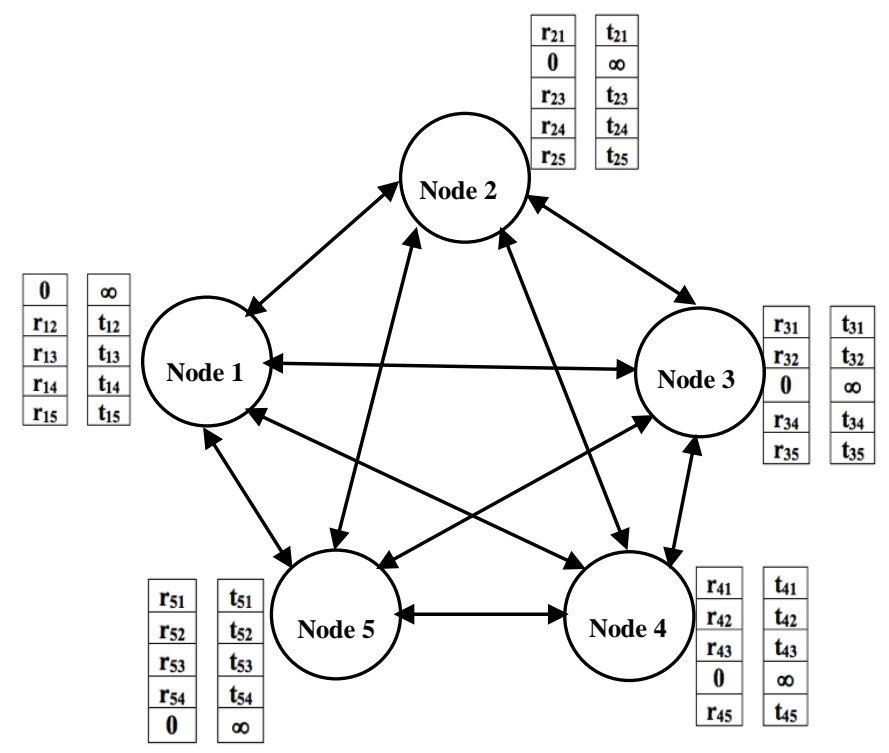

Fig. 1. A Sample of the Proposed Network Model. 
The tables are updated every $T$ time period where $T$ is inversely proportional to the amount of data transmitted across the whole network. Each node is allowed to define its own $T$ time period in order to provide reasonable parameters of its $r_{i j}$ and $t_{i j}$. As a definition, the average transmission rate between a node and itself is zero $\left(r_{i j}=0, i=j\right)$ and the number of times a node used itself for data sending is infinity $\left(t_{i j}=\right.$ $\infty, i=j)$.

In the proposed network model, mobile nodes communicate with their neighboring node to exchange the average transmission rates and the number of time that each node has been used for data transmission to decide which node the data will be sent through. In order to prioritize the importance of the two parameters in the proposed scheme, we introduce relative weights that is used to tradeoff between them based on the user demand and network condition. The purpose of the relative weights is providing a flexibility to the network model and comply with network demands. For a given network model with $N$ number of nodes, let $\alpha$ is a relative weight of the average transmission rates $r$ and $\beta$ is the relative weight of number of times a node used its neighbor for data transmission $t$. The characteristic function $f_{T}$ is defined as:

$f_{T}(N)=\alpha \cdot r+\beta \cdot t$

where $0<\alpha<1,0<\beta<1$ and $\alpha+\beta=1$

Due to the mobility in MANETs, it is important to keep tracking and monitoring of the neighboring nodes at any time. The relative weights provide the network the flexibility and reliability of prioritizing the important parameter based on the network condition. The relative weight $\alpha$ helps to provide the maximum weight of the transmission rate parameter, while $\beta$ helps to provide the power usage of a given node.

\section{Proposed SCHEME Algorithm}

In this section, we introduce the algorithm of our proposed model. Several assumptions are considered in order to operate the algorithm. For mobile nodes, we assume that there $N$ rational mobile nodes, all nodes have the same battery capacity, individual nodes have weak chance to survive alone and all nodes are able to create two tables one for the neighbors average transmission rate and the other one is for the neighbors' power. For the network, we assume that network is not of a hierarchical type and the goal of the cooperative game is to form a stable network.

The algorithm below describes the data transmission of a node after selecting the proper neighboring node within a time slot $T$. The time slot $T$ and the weight parameters $\alpha$ and $\beta$ can be changed according to the network requirements. Initially, the weight parameters $\alpha$ and $\beta$ are assigned equally to all coalition nodes. Then each node checks the transmission rate of its neighbor nodes and the number of times that a node has been used for data transmission. If node $i$ selects a specific node $j$, then node $i$ announces to all neighboring nodes, otherwise it sends nothing. When node $i$ uses node $j$, all neighboring nodes updates the power usage table. The algorithm is repeated every $T$ time slot to keep tracking the nodes power usage.
1. Assign values for $\alpha$ and $\beta$

2. Start for all nodes

3. Node $i$ checks its transmission rate table and power table to assign Neighbor node $j$ for data sending according to equation (1)

4. If node $i$ used node $j$ for sending data then 4.1. Announce a node power usage for this specific node 4.2. Else find another node 4.3. End if

5. Store this power usage value in the power table

6. Share power usage value with neighbors at every timeslot.

7. All nodes continue to update their power and transmission rates tables.

\section{Simulation AND RESUlts}

The proposed approach is implemented using NS-2 Simulator (NS-2, 2021). The parameters for the simulation is shown in Table I where several test cases are produced based on the variations in number of neighboring nodes, weight parameters, network delay, network overhead, transmission rate and power consumption. Some of test cases show the effect of increasing the neighboring nodes with respect to their life time while others shows the effect of varying of the $\alpha$ and $\beta$ weight parameters of the characteristic function with respect to, the network delay and overhead. A sample of 50, 80, 100 and 150 as size of coalition nodes are used in simulating the test cases. The following sections describe the results of the simulated test cases.

TABLE I. PARAMETERS FOR SIMULATION

\begin{tabular}{|l|l|}
\hline Parameters & Level \\
\hline Area & $4000 * 3200$ \\
\hline Speed & $18 \mathrm{~m} / \mathrm{s}$ \\
\hline Radio range & $800 \mathrm{~m}$ \\
\hline MAC & 802.11 \\
\hline Simulation time & $1200 \mathrm{~s}$ \\
\hline Size of the coalition nodes & $50,80,100$ and 150 \\
\hline Network interface type & Wireless \\
\hline Channel type & Wireless channel \\
\hline Transmission rate & Vary \\
\hline Initial energy & 100 Joule \\
\hline Transmission power & 0.4 Joule \\
\hline Receiving power & 0.05 Joule \\
\hline
\end{tabular}

\section{A. Energy Consumption}

In this section, the test case focuses on the amount of remaining energy based on specific node. The selected node is tested based on different coalition sizes. For fairness and precise results, we assume equal energy consumption for every transmitted data. Fig. 2 describes the percentage of the remaining energy based on the coalition sizes defined in Table I. The figure shows that by increasing the size of coalition, the energy consumption for a given node is 
decreased (the remaining battery energy are 51\%, 60\%, 66\% and $80 \%$ for coalition sizes 50, 80, 100 and 150 , respectively). This is because the number of neighboring node increases, so the number of times a node is used for data transmitting is reduced.

Fig. 3 shows a comparison of the overall remaining energy (in percent) of a network before and after using our scheme for a sample of 150 coalition size. As the time passes, the saving of coalition energy is improved by the proposed scheme (energy saved by $19 \%$ at $60 \mathrm{sec}$. and $28 \%$ at $120 \mathrm{sec}$ ).

\section{B. Weight Parameters}

In this section, the test cases focus on $\alpha$ and $\beta$ weight parameters. These parameters allow the network to monitor the bias towards either the data transmission rate or the energy consumption of mobile nodes. The default situation is to have an equal value of $(\alpha=\beta=0.5)$. We investigate the effects of changing the weight parameters on network overhead and number of dead nodes.

1) Network throughput and delay: Fig. 4 describes the relationship between the network throughput and the coalition size. The test case uses a sample of 50, 80, 100 and 150 coalition sizes. Initially all coalition sizes have an equal; and as time passes the throughput of higher coalition size becomes greater. At time $=60 \mathrm{sec}$, throughput of 50 coalition size is $51 \%$ while it is $68 \%$ for 150 coalition size. At time $=120 \mathrm{sec}$, throughput of 50 coalition size is $83 \%$ while it is $98 \%$ for 150 coalition size.

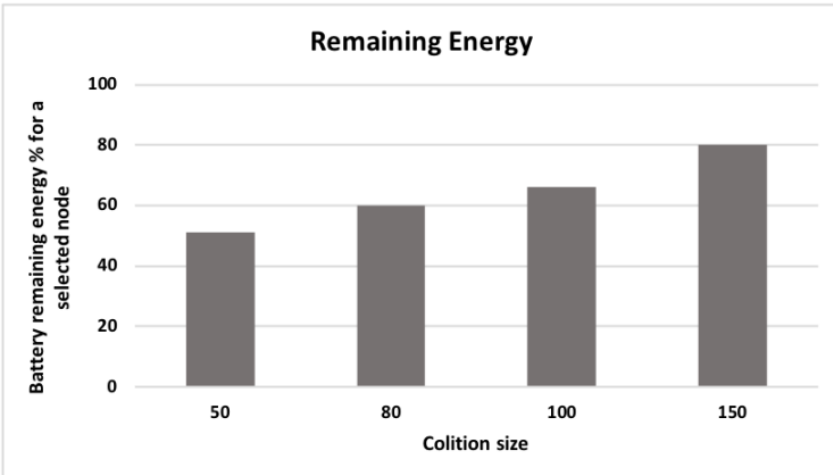

Fig. 2. Percentage of the Remaining Energy for a Coalition of Nodes.

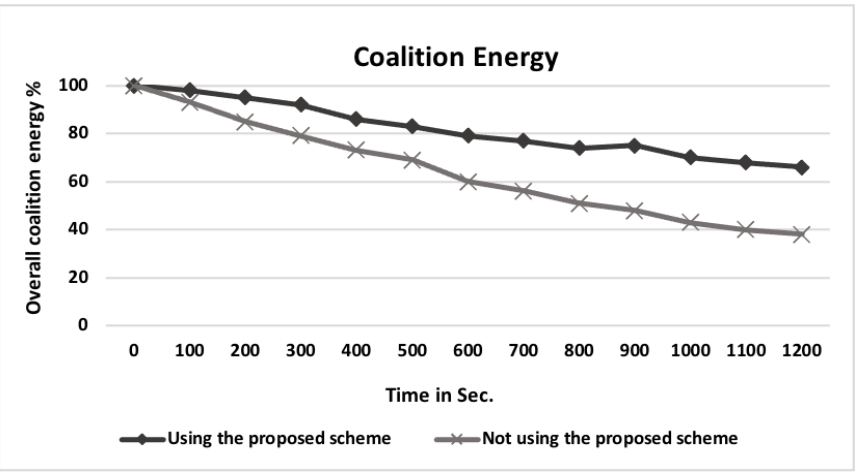

Fig. 3. Overall Coalition Energy Percentage with and without using the Proposed.

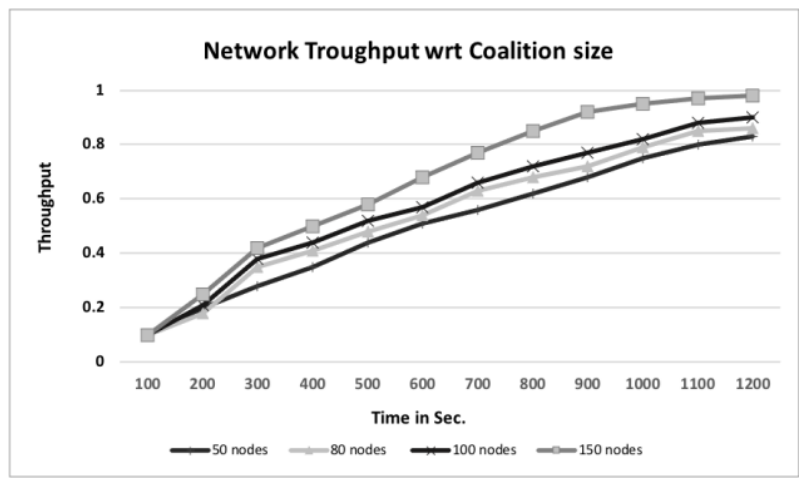

Fig. 4. Throughput for different Coalition Sizes.

The same time applies in Fig. 5 where the relationship is described between the network delay and the coalition size. The test case shows that the network delay decreases as the coalition size increases. Initially, the network delay of 50 coalition size equals to $80 \%$, while it is $27 \%$ for 150 coalition size. At time $=10$, the network delay equals to $15 \%$ for the 50 coalition size and $1 \%$ for 150 coalition size.

2) Dead nodes and $\alpha$ weight parameter: Fig. 6 describes the relationship between the weight of the average transmission rate $(\alpha)$ and the dead nodes. The test cases of different coalition sizes show that as the weight parameter $\alpha$ increases the number of dead nodes increases. This is because as the average transmission rate increases, the power consumption of mobile nodes increases and that would increase the chance of dead nodes. Also, the test cases show that the chances of having dead nodes decreases as the coalition size increases.

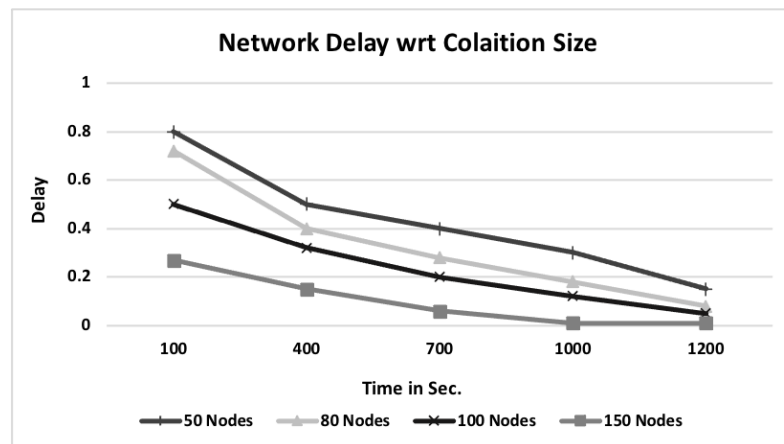

Fig. 5. Delay for different Coalition Sizes over time.

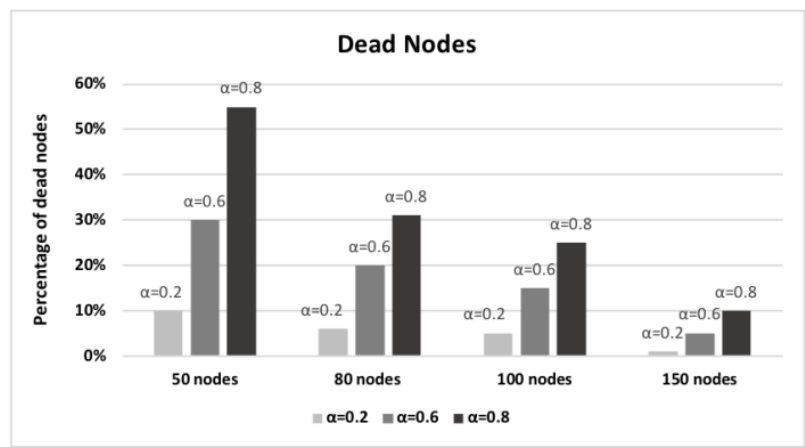

Fig. 6. Number of Dead Nodes with different Weights of $\propto$. 
3) Network overhead and $\beta$ weight parameter: Fig. 7(a), 7(b) and 7(c) describe the relationship between the network overhead and the weight of number of times a node used its neighbor for data transmission. The network overhead is a reflection of network delay. The $\beta$ weight parameter monitors the nodes remaining power battery. The test case in Fig. 7(a) shows the overhead of four coalition sizes when $\beta=0.8$. Initially all coalition size have the same overheads. At time $=$ $60 \mathrm{sec}$ the overhead of less coalition size becomes greater, $38 \%$ for 50 coalition size and $20 \%$ for 150 coalition size. While at time $=160 \mathrm{sec}$, it is $53 \%$ for 50 coalition size and $29 \%$ for 150 coalition size. The same thing applies to Fig. 7(b) and 7(c) where $\beta=0.6$ and $\beta=0.2$, respectively. Notice that as $\beta$ increases for the same coalition size, the overhead increases. As an example, for 50 coalition size, the overhead equals $13 \%$ when $\beta=0.2$ and it is $45 \% \beta=0.6$ and it is $53 \%$ when $\beta=0.8$.

\section{Scheme Evaluation}

In this section, we compare the proposed scheme with respect to the other schemes within the same category as described in Section 2. The comparison described in Table II

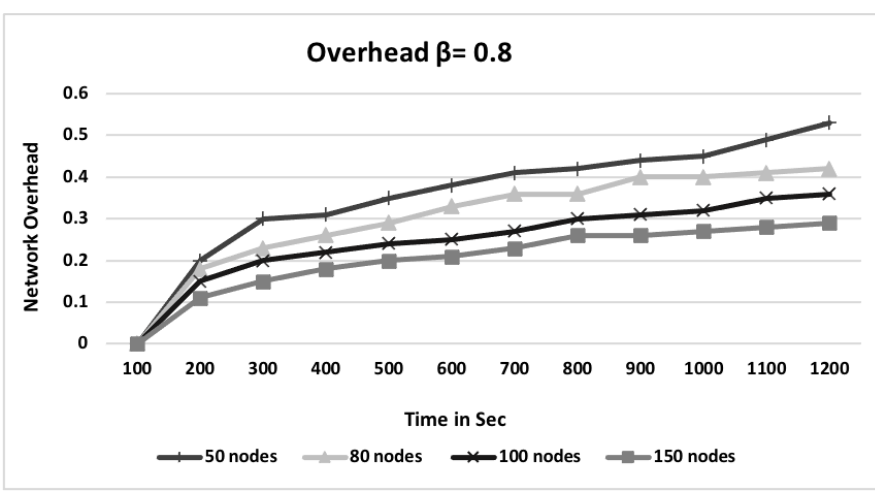

(a). Network Overhead with $\beta=0.8$. covers five criteria: power model, discovery method, merits, route metrics and performance metrics. Some of them are defined in [11]. The power model can be constant when the link cost is fixed regardless of the source-destination distance or variable when the link cost depends on the sourcedestination distance. The routing scheme uses single-hop discovery method when its procedure rely on the neighboring nodes or multi-hop when the procedure relies on the overall path from the source to destination.

Table II summarizes a comparison between the related schemes and the proposed one. Some of the features are similar in all routing schemes. However, our proposed scheme differs in the discovery method since it uses cascaded singlehop. The cascaded single-hop supports the optimal selection of routing path at every source-destination hop with minimum transmission overhead. Also, the algorithm of the proposed scheme is simple since the selection of the routing path depends on two metrics, the average transmission rate for the neighboring nodes and number of times the neighboring node is used for data transmission during the network life cycle.

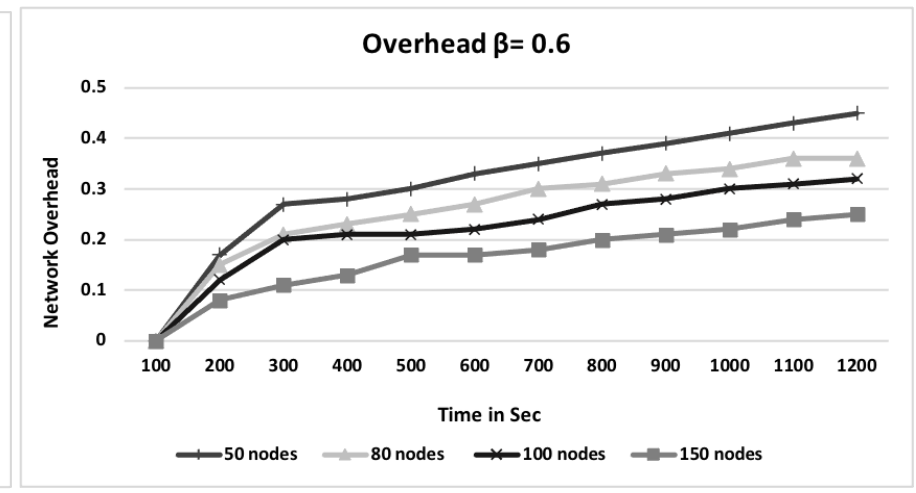

(b). Network Overhead with $\beta=0.6$.

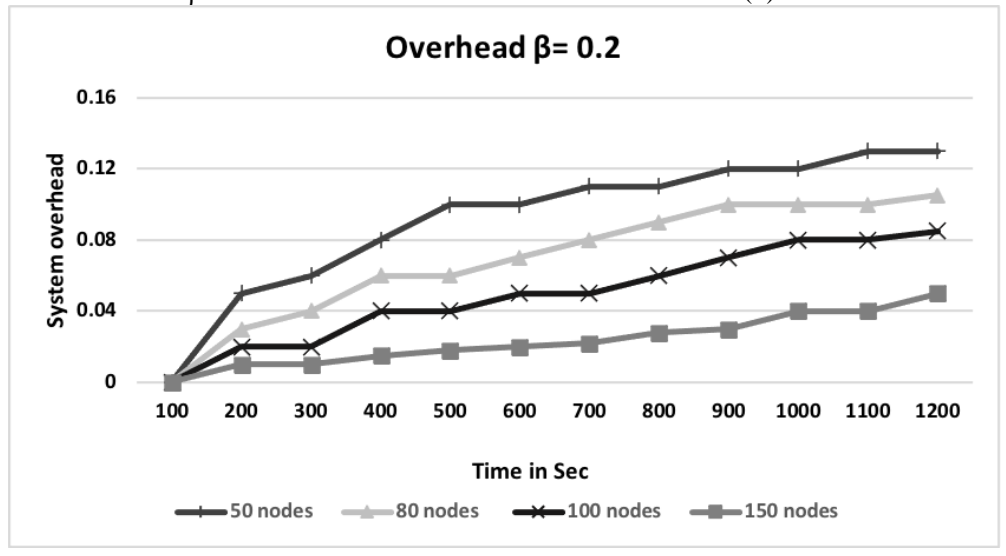

Fig. 7. (c). Network Overhead with $\beta=0.2$. 
TABLE II. COMPARISON BETWEEN THE PROPOSED SCHEME AND RELATED SCHEMES

\begin{tabular}{|c|c|c|c|c|c|}
\hline Routing scheme & Power Model & Discovery Method & Merits & Route Metrics & Performance Metrics \\
\hline Proposed approach & Variable & Cascaded single-hop & $\begin{array}{l}\text { Simple algorithm, Direct } \\
\text { trade-off between the power } \\
\text { consumption and number of } \\
\text { transmissions }\end{array}$ & $\begin{array}{l}\text { Number of } \\
\text { transmissions of a } \\
\text { node and number of } \\
\text { times the neighboring } \\
\text { node }\end{array}$ & $\begin{array}{l}\text { Network life time, normalized } \\
\text { residual energy and minimum } \\
\text { transmission over Head }\end{array}$ \\
\hline FAR [15] & Constant & Multi-hop & $\begin{array}{l}\text { Maximize the network life } \\
\text { time }\end{array}$ & Link and node costs & $\begin{array}{l}\text { Network life time and normalized } \\
\text { residual energy }\end{array}$ \\
\hline OMM [16] & Variable & Multi-hop & $\begin{array}{l}\text { Maximizes the network life } \\
\text { time }\end{array}$ & $\begin{array}{l}\text { Max-Min Residual } \\
\text { power }\end{array}$ & $\begin{array}{l}\text { Maximum of transmitted messages } \\
\text { and ratio the optimal solution, }\end{array}$ \\
\hline MER [17] & Variable & Multi-hop & $\begin{array}{l}\text { Direct tradeoff between } \\
\text { routing overhead and lower } \\
\text { energy consumption }\end{array}$ & Minimum hop route & Normalized energy \\
\hline DEAR [18] & Variable & Multi-hop & $\begin{array}{l}\text { Based on energy-aware } \\
\text { devices }\end{array}$ & Device-aware & Network lifetime \\
\hline PAR [19] & Variable & Multi-hop & $\begin{array}{l}\text { Selection of the less } \\
\text { congested path and energy } \\
\text { efficient. }\end{array}$ & Link status ratio & $\begin{array}{l}\text { Network energy consumption, } \\
\text { number of failed Nodes and } \\
\text { average of residual energy }\end{array}$ \\
\hline MST-BDD [20] & Variable & Multi-hop & $\begin{array}{l}\text { Simple algorithm, Decreases } \\
\text { the network power } \\
\text { transmission }\end{array}$ & Minimum SPT & Time complexity \\
\hline AODV_RR [21] & Variable & Multi-hop & $\begin{array}{l}\text { Lower overall Energy } \\
\text { Consumption and } \\
\text { communication overhead }\end{array}$ & $\begin{array}{l}\text { Received signal } \\
\text { strength }\end{array}$ & $\begin{array}{l}\text { Multiple of QoS such as } \\
\text { throughput, delay, amount of } \\
\text { overhead }\end{array}$ \\
\hline ELRPP [22] & Variable & Multi-hop & Improves QoS & $\begin{array}{l}\text { Link availability and } \\
\text { existing energy }\end{array}$ & $\begin{array}{l}\text { Multiple of QoS such as } \\
\text { throughput, delay, total energy } \\
\text { consumption, drop ratio and } \\
\text { execution time }\end{array}$ \\
\hline
\end{tabular}

\section{CONCLUSION AND FUTURE}

In this paper, we propose a new scheme based on transmission power control-based approach to provide a balance between the transmission rates and power consumption of mobile nodes. The scheme uses a cascaded single-hop to discover the utilization and the average transmission rate of the neighboring node. The scheme algorithm selects the routing path by creating routing tables for each neighboring node. The table contains the average transmission rate for the neighboring nodes and number of times the neighboring node is used for data transmission. The proposed approach affects the overall network lifetime by increasing the battery power of the nodes and decreasing the power consumption in the network. Through simulating several test cases, we discussed how nodes can select a proper node for transmitting data using a unique mechanism that keeps track of the transmission rates and energy consumption of individual nodes in the network. The simulation showed how varying characteristics function weight parameters can affect the network sensitive parameters.

As a future, we are planning to study the model to that it can be scaled up to include thousands of nodes and consider the features of the proposed scheme to operate in a bigger coalition size; also, to study the response of the algorithm under different types of attacks.
REFERENCES

[1] S. Plass, F. Clazzer, B. Fritz, I. Yasrine, M. Maurizio, "Maritime communications - Identifying current and future satellite requirements \& technologies", 20th Ka and Broadband Communications, Navigation and Earth Observation Conference, Italy, 2014.

[2] G. Bonanno, "Game Theory: An open access textbook with 165 solved exercises", Open Access, 2015.

[3] N. Karayiannis, S. Nadella, "Power-conserving routing of ad hoc mobile wireless networks based on entropy constrained algorithms", Ad Hoc Networks, Vol. 4, No. 1, pp. 24-35, 2006.

[4] J. Zhu, B. Bensaou, F. Nait-Abdesselam, "Power control protocols for wireless ad hoc networks"', In Algorithms and Protocols for Wireless and Mobile Ad Hoc Networks, John Wiley \& Sons Inc., pp.315-352, 2008.

[5] S. Giordano, I. Stojmenovic, L. Blazevic, "Position based routing algorithms for ad hoc networks: A taxonomy", Ad Hoc Wireless Networking, Vol. 14, pp. 103-136, 2004.

[6] A. Al Sharah, M. Alhaj, "Using Hand-shake Transmission Rate Adaptation Scheme in MANET", IJCSNS International Journal of Computer Science and Network Security, Vol. 20, No. 2, 2020.

[7] B. Devika, P. N. Sudha, "Power optimization in MANET using topology management", Engineering Science and Technology, an International Journal 23(3) pp 565-575, 2020.

[8] J. Naser, A. Kadhim, "Multicast routing strategy for SDN-cluster based MANET", International Journal of Electrical \& Computer Engineering (2088-8708), 10, 2020

[9] S. Kumar, D. Sinha, V. Kumar, "An Approach to Improve Lifetime of MANET via Power Aware Routing Protocol and Genetic Algorithm", 2nd International Conference on Innovative Mechanisms for Industry Applications (ICIMIA), pp. 550-553, IEEE, 2020. 
[10] D. Sinwar, N. Sharma S. Maakar, S. Kumar, "Analysis and comparison of ant colony optimization algorithm with DSDV, AODV, and AOMDV based on shortest path in MANET", Journal of Information and Optimization Sciences, 41(2), pp. 621-632, 2020.

[11] W. Jabbar, M. Ismail, R. Nordin, S. Arif, "Power- efficient routing schemes for MANETs: a survey and open issues", Wireless Network Vol. 23, pp. 1917-1952, 2017.

[12] Y. Ko, N. Vaidya, "Location-Aided Routing (LAR) in mobile ad hoc networks", Wireless Networks, Vol. 6, No 4, pp. 307-321,2000.

[13] A. Al Sharah, M. Alhaj, M. Hassan, "Selfish Dynamic Punishment Scheme: Misbehavior Detection in MANETs Using Cooperative Repeated Game", IJCSNS International Journal of Computer Science and Network Security, Vol. 20, No. 3, 2020.

[14] The Network Simulator - NS-2, [Online] https://www.isi.edu/nsnam/ns/ (Accessed 15/1/2021).

[15] J. Chang, L. Tassiulas, "Energy conserving routing in wireless ad-hoc networks", In Proceedings-IEEE INFOCOM, Vol. 1, pp. 22-31, 2000.

[16] Q. Li, J. Aslam, D. Rus, "Online power-aware routing in wireless ad-hoc networks", In Proceedings of the 7th annual international conference on Mobile computing and networking, pp. 97-107, 2001.

[17] S. Doshi, T. Brown, "Minimum energy routing schemes for a wireless ad hoc networks", In Proceedings of the Conference on Computer Communications, Vol. 2, 2002.

[18] A. Avudainayagam, W. Lou, Y. Fang, "DEAR: A device and energy aware routing protocol for heterogeneous ad hoc networks", Journal of Parallel and Distributed Computing, Vol. 63, No. 2, pp. 228-236, 2003.

[19] V. Rishiwal, M. Yadav, S. Bajapai, S. Verma, "Power aware routing in ad hoc wireless networks", Journal of Computer Science and Technology, Vol. 9, No. 2, pp. 101-109, 2009.

[20] C. Yanez-Marquez, I. Lopez-Yanez, O. Camacho-Nieto, A. ArguellesCruz, "BDD-based algorithm for the minimum spanning tree in wireless ad-hoc network routing", IEEE on Latin America Transactions, Vol. 11, No. 1, pp. 600-601, 2013

[21] V. Lalitha, R. Rajesh, "AODV_RR: A maximum transmission range based ad hoc on-demand distance vector routing in MANET", Wireless Personal Communications, Vol. 78, No. 1, pp. 491-506. doi:10.1007/s11277-014-1763-6, 2014.

[22] J. Katiravan, D. Sylvia, D. Rao, "Energy efficient link aware routing with power control in wireless ad hoc networks", The Scientific World Journal, 2015

[23] S. Kannan, A. Rajaram, "QoS Aware Power Efficient Multicast Routing Protocol (QoS-PEMRP) with Varying Mobility Speed for Mobile Ad Hoc Networks", Iinternational Journal of Computer Applications, Vol. 60, No. 18, 2012.
[24] C. Papageorgiou, P. Kokkinos, M. Varvarigos, "Energy-efficient multicasting in wireless networks with fixed node transmission power", Proceedings of the International Conference on Wireless Communications and Mobile Computing: Connecting the World Wirelessly, Germany, 2009.

[25] A. Khan, Q. Sun, Z. Mahmood, A. Ghafoor, "Energy Efficient Partial Permutation Encryption on Network Coded MANETs", Journal of Electrical and Computer Engineering, Vol. 2017, 2017.

[26] M. Rahman, "An teeiciffE Position based Power Aware Routing Algorithm in Mobile Ad-hoc Networks", I. J. Computer Network and Information Security, 2016.

[27] S. Ghode, K. Bhoyar, "NEMA: Node Energy Monitoring Algorithm for Zone Head Selection in mobile ad-hoc network using residual battery power of node", International Conference on Wireless Communications, Signal Processing and Networking (WiSPNET), India, 2016.

[28] M. Hassan, R. Muniyandi, "An Improved Hybrid Technique for Energy and Delay Routing in Mobile Ad-Hoc Networks", International Journal of Applied Engineering Research, Vol. 12, No. 1, pp. 134-139, 2017.

[29] T. Lu, J. Zhu, "Genetic algorithm for energy-efficient QoS multicast routing", IEEE Communications Letters, Vol. 17, No. 1, pp. 31-34, 2013.

[30] P. Kamboj, A. Sharma, "Power aware multicast reactive routing protocol (PAMRRP)", IJCSNS International Journal of Computer Science and Network Security, Vol. 8, No. 8, 2008.

[31] S. Zhao, L. Tan, J. Li, "A distributed energy efficient multicast routing algorithm for WANETs "International Journal of Sensor Networks, 2007.

[32] N. Papanna, A. Reddy, M. Seetha, "EELAM: Energy efficient lifetime aware multicast route selection for mobile ad hoc networks", Applied Computing and Informatics, Vol. 15, No. 2, pp. 120-128, 2019.

[33] F. Sarkohaki,, R. Fotohi, and V. Ashrafian, "An Efficient Routing Protocol in Mobile Ad-hoc Networks by using Artificial Immune System", International Journal of Advanced Computer Science and Applications (IJACSA), Vol. 8, No. 4, pp. 554-561, 2020.

[34] N. Ray, A. Turuk, "A Hybrid Energy Efficient Protocol for Mobile Ad Hoc Networks", Journal of Computer Networks and Communications, Vol. 2016, Article ID 2861904, 2016.

[35] S. Alani, Z. Zakaria, H. Lago, "A new energy consumption technique for mobile Ad-Hoc networks" International Journal of Electrical and Computer Engineering (IJECE), Vol. 9, No. 5, pp. 4147 4153, 2019.

[36] B. Bhatia, M. Soni, P. Tomar, "Extended Bandwidth Optimized and Energy Efficient Dynamic Source Routing Protocol in Mobile Ad-hoc Networks", International Journal of Electrical and Computer Engineering (IJECE), Vol. 8, No. 3, pp. 1460-1466, 2018. 